\title{
Prevalence of vulval lichen planus in a cohort of women with oral lichen planus: an interdisciplinary study
}

\author{
Mother and Child Department, Gynaecology and Obstetrics Institute, \\ * Sector of Oral Medicine, Department of Oral Sciences, \\ $\dagger$ Institute of Pathology, \\ \$Department of Quantitative Methods for the Human Sciences, University of Palermo, Palermo, Italy
}

P. Belfiore, O. Di Fede, ${ }^{\star}$ D. Cabibi, $\uparrow$ G. Campisi,* G.S. Amarù, S. De Cantisł and E. Maresi†

\section{Summary}

\section{Correspondence \\ Pina Belfiore, Istituto di Ginecologia e Ostetricia Universitá degli Studi di Palermo. Via del Vespro, 12990127 Palermo, Italy. \\ E-mail: pinabelfiore@tiscali.it}

\section{Accepted for publication \\ 18 May 2006}

\section{Key words}

genital lichen planus, oral lichen planus, vulval lichen planus

\section{Conflicts of interest}

None declared.
Background Lichen planus (LP) is a mucocutaneous inflammatory dermatosis that frequently involves the oral and genital mucosae. Patients with LP affecting these sites are often seen by oral medicine specialists or gynaecologists who work in isolation and depend heavily on histopathologists to help them in confirming the diagnosis. There are few studies in the literature combining the experiences of these specialists who share the care of patients with both oral and genital LP.

Objectives To estimate the prevalence of vulval LP (VLP) in a cohort of patients with histologically confirmed oral LP (OLP).

Methods The study group consisted of 42 women histologically diagnosed with OLP. The mean age was 60.5 years (range 27-81). They underwent genital examination, colposcopy and vulvoscopy. For the histological confirmation of clinical VLP biopsies were performed whenever a clinical lesion was found. Oral and genital biopsy specimens were processed through histological and immunohistochemical staining. Histological diagnoses of LP were made according to the modified World Health Organization histopathological criteria proposed by van der Meij and van der Waal for the diagnosis of OLP, and extended to VLP. Patients with clinical evidence, but without the histological confirmation of OLP and VLP, were excluded from the study group.

Results Thirty-two vulval and one vaginal biopsy specimens were obtained. Histological diagnoses were confirmed in 24 of $32(75 \%)$ patients who underwent a vulval biopsy: these represent 57\% (24 of 42) of the study group. Of the 12 patients free of symptoms such as itching, burning and dyspareunia, but with clinical vulval lesions, 11 (92\%) had histological confirmation of VLP. Vulval lichen sclerosus was ascertained in five of $32(16 \%)$ cases.

Conclusions This study showed a $57 \%$ prevalence of VLP in selected patients with OLP. The high prevalence of VLP of $92 \%$ in the women who were free of vulval symptoms confirmed the usefulness of this careful integrated approach.
Lichen planus (LP) is an inflammatory dermatosis which may involve the skin, oral and genital mucous membranes and several other sites. Oral lesions occur as the only manifestation of LP in $15-35 \%$ of patients, but up to $65 \%$ of patients with classic cutaneous disease have oral involvement. ${ }^{1}$ Vulval LP (VLP) may often be associated with desquamative inflammatory vaginitis (DIV), a condition that is secondary to any erosive or blistering epithelial disease that occurs in the vagina. ${ }^{2}$

Approximately $25 \%$ of men with typical cutaneous LP exhibit genital lesions, but the frequency of genital LP in women is less known. ${ }^{2,3}$ A retrospective study showed a VLP frequency of $3 \cdot 7 \%$ in the population attending a vulval clinic during a 13-year period. ${ }^{4}$ The vulvovaginogingival syndrome (VVGS) was described as a distinctive pattern of erosive plurimucosal $L P^{5}$ and is a clinical triad of vulval, vaginal and gingival LP.

This knowledge and the lack of systematic study on VLP prompted us to carry out an interdisciplinary study on a selected population integrating the competences of an oral practitioner, pathologists and a vulvologist/gynaecologist. The aim 
of this study was to estimate the prevalence of VLP in a cohort of patients with histologically confirmed oral LP (OLP).

\section{Patients and methods}

Fifty-four women with clinical evidence of OLP were enrolled in this study between October 2001 and July 2004. There was no histological confirmation of OLP in 12 patients, and they were therefore excluded from the study (Fig. 1). The study group thus consisted of 42 women with histologically diagnosed OLP. Their mean age was 60.5 years (range 27-81). Each patient gave her informed consent to the study and to the use of personal data.

\section{Clinical examination}

All examinations were carried out by one of the authors (O.D.F.) trained to recognize oral mucosal disorders. Burning was the only oral symptom reported. The clinical presentation of OLP lesions (Fig. 2) was classified into three forms: reticular, erosive/atrophic and ulcerative. In all patients the clinical diagnoses were confirmed by histological analyses obtained by means of biopsies. Histological features of OLP, as well as differential diagnoses with oral lichenoid reactions, were as reported in recent literature. ${ }^{6,7}$

Genital examination was carried out by a trained vulvologist/gynaecologist (P.B.). Vulval symptoms were described as itching, burning or dyspareunia. Clinical examination of the

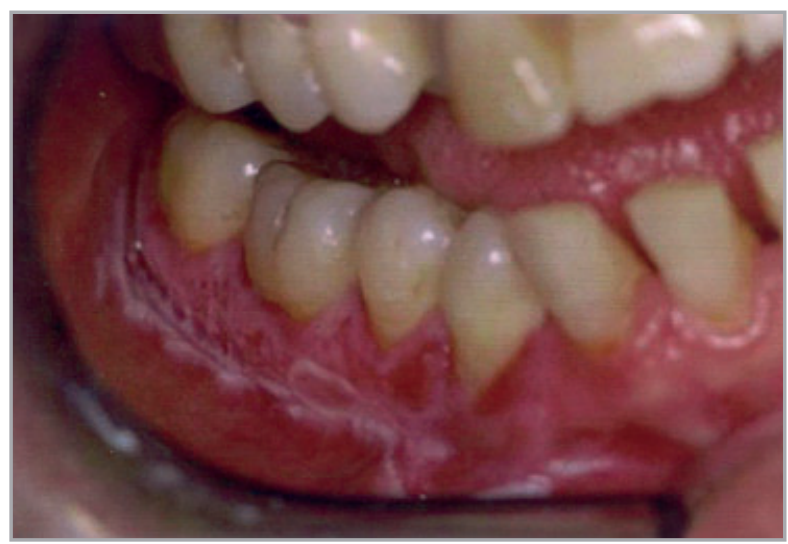

Fig 2. Oral lichen planus lesion showing white reticulate area on the lower gingiva.

vulva included inspection of skin colour and surface, and palpation for evaluating the texture and elasticity. The main clinical patterns of lesions recognized were the classical papular form (Fig. 3), the erosive/atrophic form (Fig. 4) and the hypertrophic form (Fig. 5).

Erythema, erosions of the wall and increased vaginal discharge were the clinical features of DIV associated with the vulval symptoms mentioned above. Vaginal secretions microscopically show an increase in white blood cells, a lack of lactobacilli and a high $\mathrm{pH}(6-7)$. Histology is aspecific. ${ }^{2}$ Colposcopic examination of the cervix with $5 \%$ acetic acid and

Fig 1. Flow diagram of the protocol and

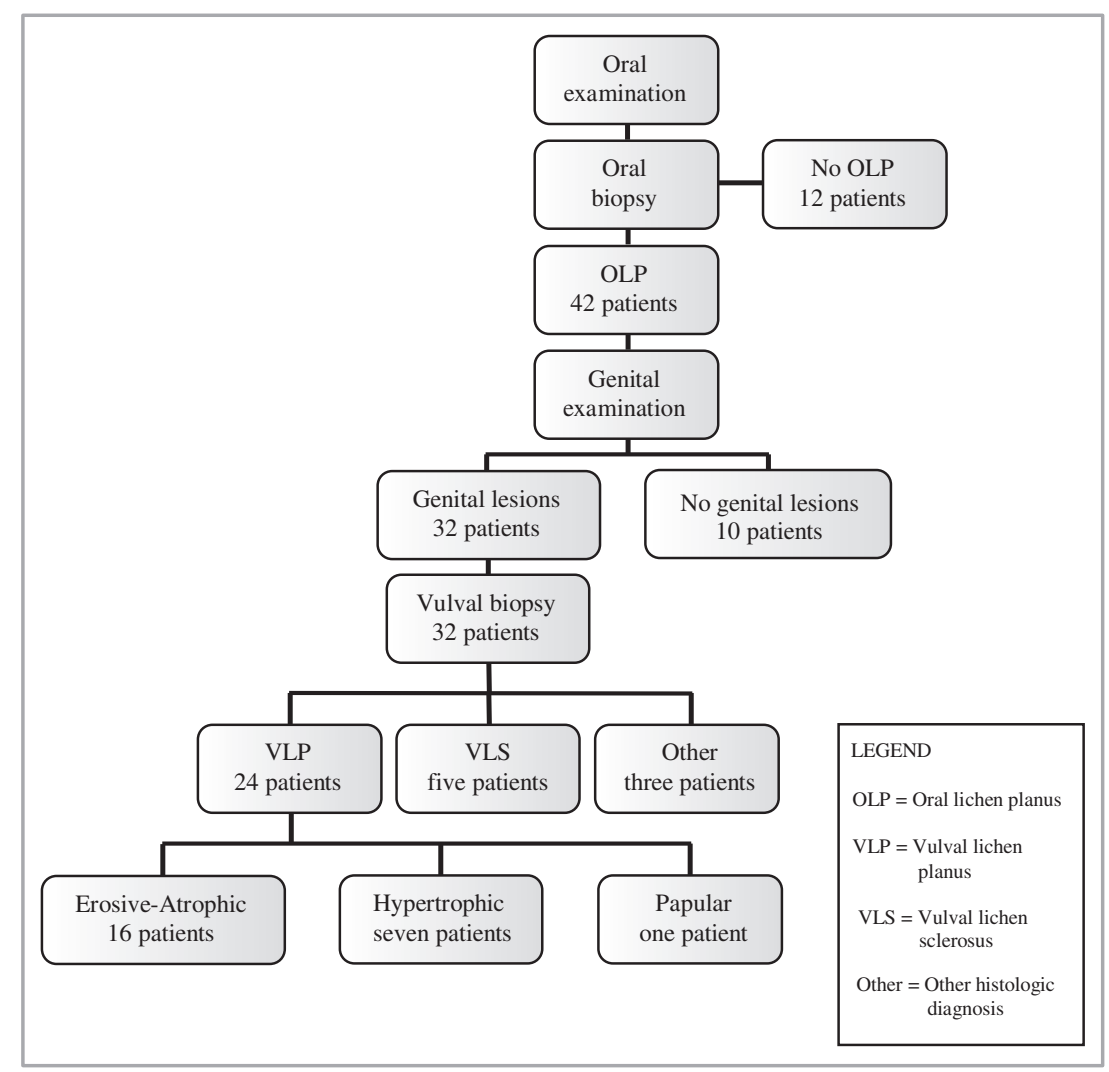

study population. 


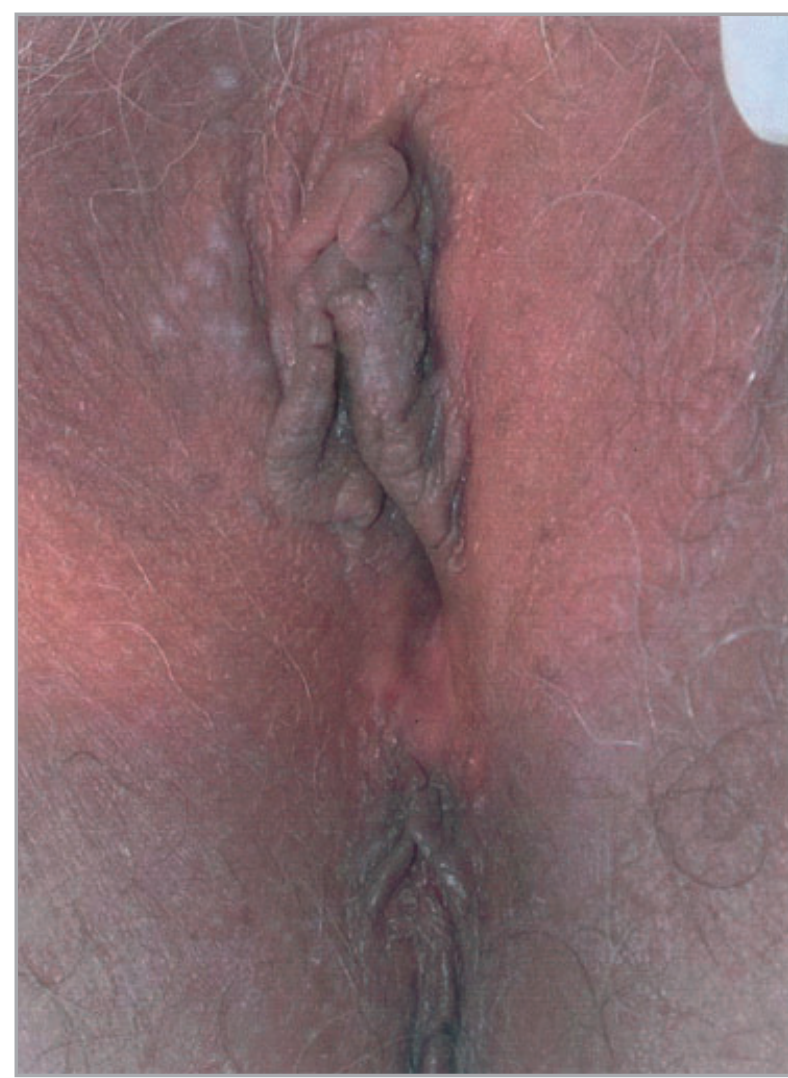

Fig 3. Classical papular vulval lichen planus with typical whiteviolaceous papules grouped on the right labium majus.

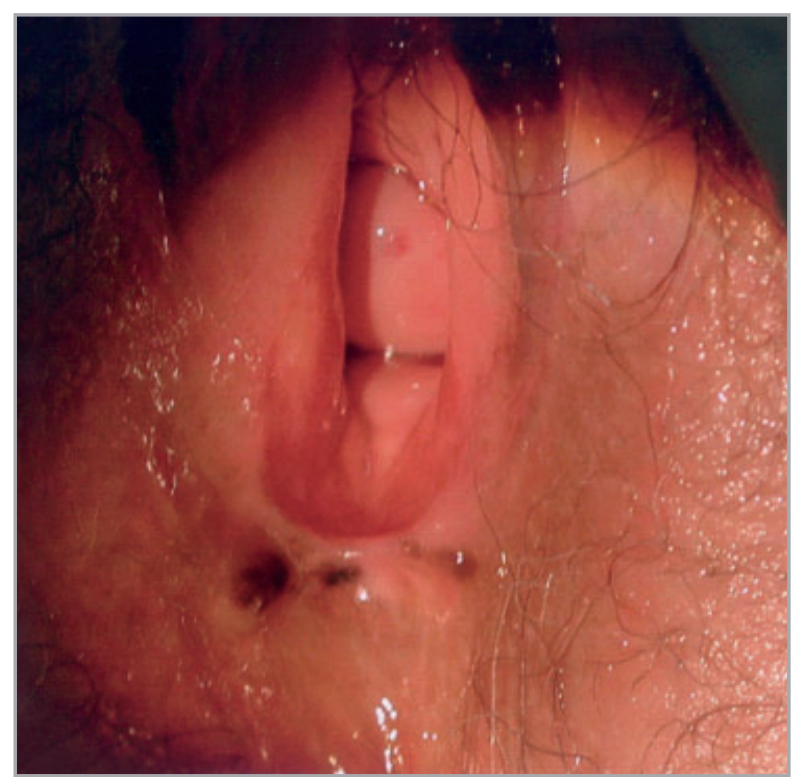

Fig 4. Erosive vulval lichen planus involving the labia minora, vestibule and frenulum, with erosion of the vaginal anterior wall and postinflammatory pigmentation on the frenulum.

iodine solution was made. For the histological confirmation of genital LP one or more biopsies were performed whenever a clinical lesion was found.

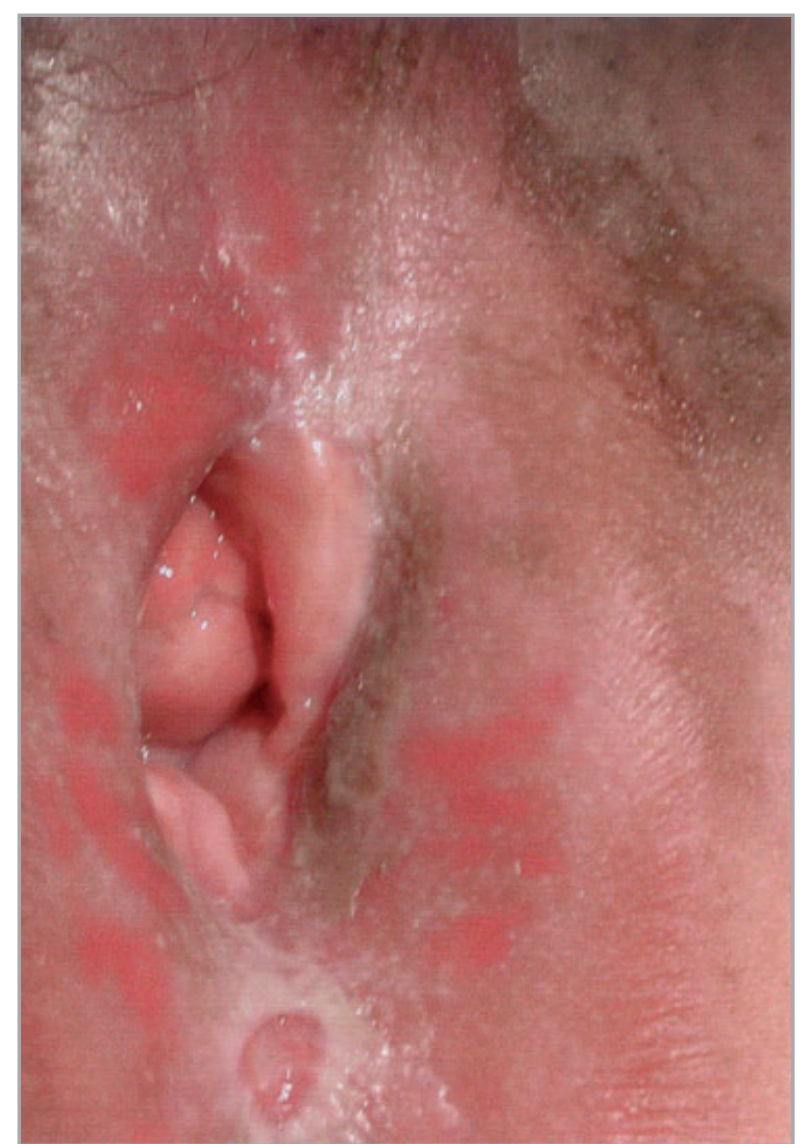

Fig 5. Hypertrophic vulval lichen planus with loss of vulval architecture, erosion, ulceration and narrowing of introitus.

\section{Histological procedures and criteria for diagnosis of oral lichen planus and vulval lichen planus}

Formalin-fixed, paraffin-embedded tissue sections were stained with haematoxylin and eosin and were observed by two pathologists separately (E.M. and D.C.). The following histological characteristics were considered: the signs of liquefaction degeneration in the basal cell layer, the presence of a well-defined band of cellular infiltration, confined to the superficial part of the connective tissue, and the strong predominance of lymphocytes in the inflammatory infiltrate (Fig. 6). The presence of each of these histological aspects was registered as follows: 0 , absent; 1 , present.

Each case was categorized as 'histologically diagnostic of OLP/VLP' only when all the aforementioned criteria were present (category 1); 'compatible with OLP/VLP' when only two aspects were present (category 2); and 'nonconsistent with OLP/VLP' when fewer than two aspects were evident (category 3 ).

For categories 1 or 2 an immunohistochemical study was performed by using the avidin-biotin-complex technique as described in the manufacturer's instructions (Universal LSAAB kit; Dako, Glostrup, Denmark). Polyclonal rabbit antihuman 


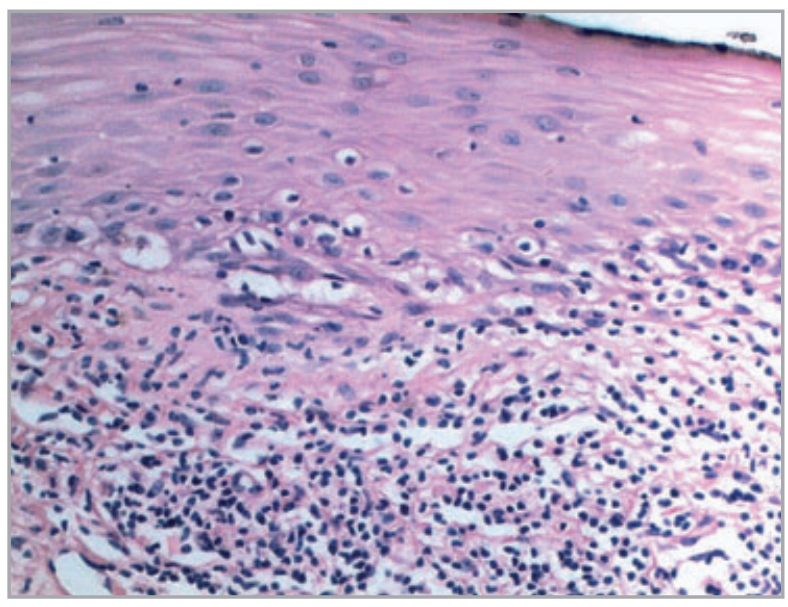

Fig 6. Vulval biopsy showing histological features of lichen planus: liquefaction degeneration in the basal cell layer and a band of cellular infiltration with predominance of lymphocytes (haematoxylin and eosin; original magnification $\times 200$ ).

CD3 (a highly specific marker for $\mathrm{T}$ cells) and monoclonal mouse antihuman CD20cy (clone L26), a marker for B cells, both obtained from Dako, were used as primary antibodies. To improve the immunohistochemical staining, tissue sections were microwaved in $10 \mathrm{mmol} \mathrm{L}^{-1}$ citrate buffer $(\mathrm{pH} \mathrm{6.0)}$ ) for heat-induced epitope retrieval before incubation with the primary antibody.

For immunohistochemical assessment, positivity for CD3 and CD20cy was qualitatively assessed and only cases with strong predominance of $\mathrm{T}$ lymphocytes were considered diagnostic of OLP/VLP. Only patients classified as histologically diagnostic of OLP/VLP (category 1) or compatible with OLP/ VLP (category 2) were included in the study group; those with clinical evidence of OLP but without histological confirmation were excluded from the study group.

\section{Results}

Of all the patients who attended the vulval clinic during the study period 42 consecutive women, histologically diagnosed as having OLP, were selected. These represented the study group. The flow diagram of our protocol for the study is represented in Figure 1.

Forty of the 42 patients (95\%) complained of oral symptoms. Genital symptoms of itching, burning and/or dyspareunia were present in 25 of the 42 patients $(60 \%)$, while the other $17(40 \%)$ were free of symptoms. The types of lesions and the main vulval areas involved are shown in Tables 1 and 2, respectively.

Clinical examination showed vulval lesions in 32 of 42 (76\%) patients and vaginal localization (VVGS) in a further two of $42(5 \%)$. No cervical lesions were found. All of the 32 patients showing vulval lesions underwent a vulval biopsy; a vaginal biopsy was also performed on one of these same patients. In another case it was not possible to carry out a vaginal biopsy due to a complicated infection. Of the 32
Table 1 Types of vulval lesions $(n=32$ patients)

\begin{tabular}{lcr}
\hline Type of lesions & Number of patients & $(\%)$ \\
\hline Papule & 1 & 3 \\
Plaque & 8 & 25 \\
Atrophy & 21 & 66 \\
Reticulate & 2 & 6 \\
Erosion & 16 & 50 \\
\hline
\end{tabular}

Table 2 Sites of vulval lesions ( $\mathrm{n}=32$ patients)

\begin{tabular}{lrr}
\hline Vulval areas of lesions & Number of patients & $(\%)$ \\
\hline Labia majora & 1 & 3 \\
Labia minora & 15 & 47 \\
Clitoris & 9 & 28 \\
Frenulum & 10 & 31 \\
Perianal & 1 & 3 \\
Perineum & 2 & 6 \\
Vestibule & 4 & 12 \\
\hline
\end{tabular}

women showing vulval lesions 20 (62\%) exhibited genital symptoms while $12(38 \%)$ did not.

Histological diagnoses of VLP were obtained in 24 of 32 (75\%) patients who underwent a vulval biopsy. These represent 57\% (24 of 42) of the study group. Of the 12 patients free of symptoms but with clinical vulval lesions, 11 (92\%) had histological confirmation of VLP and one of vulval lichen sclerosus (VLS). Of the positive biopsies, seven showed a thickened epidermis.

VLS was ascertained in five of $32(16 \%)$ patients, while three of $32(9 \%)$ had other forms of dermatitis. DIV was found in 21 of $24(88 \%)$ patients with VLP and in all forms of VVGS.

\section{Discussion}

LP is an inflammatory dermatosis which may involve the skin, oral and genital mucous membranes at several sites either simultaneously $^{8}$ or at different times. The exact incidence of the disease is unknown and its determination is complicated by the fact that this dermatosis shows a wide morphological range of clinical forms, involving one or more sites, and therefore requiring a histological diagnostic confirmation. LP is a relatively common disease for dermatologists and oral practitioners, but is often not recognized or is reported only sporadically by gynaecologists and other specialists. ${ }^{1,9,10}$ The relatively low frequency of genital LP, together with lack of information in the literature, prompted us to perform a cohort study on a selected population with OLP, integrating the competences of a vulvologist/gynaecologist, an oral medicine practitioner and two pathologists.

In the practice of a vulval clinic at the University of Turin the genital involvement of LP histologically diagnosed was 
reported in $3 \cdot 7 \%$ among 3350 women given a vulval biopsy. ${ }^{4}$ In the largest series published, genital involvement was estimated in approximately $25 \%$ of 723 patients with OLP. ${ }^{10} \mathrm{~A}$ recent report described patients (both men and women) with the unique chronic orogenital variant of erosive OLP. ${ }^{6}$ In the present study, the $57 \%$ prevalence of VLP among a cohort of 42 patients is clearly higher than expected, based on the few reports available in the literature.

Genital LP is a dramatic clinical presentation when the lesions present all symptoms and simultaneously involve the vulva and vagina. Most patients experience dyspareunia so severe as to preclude normal sexual activity. ${ }^{5}$ In our experience $60 \%$ of the study group had genital symptoms compared with $95 \%$ with oral symptoms. None the less, in a number of patients symptoms were absent or not yet evident. In a series of 122 patients with the VVGS of erosive LP, genital symptoms emerged after oral symptoms in $33.6 \%{ }^{6}$ The fact that $40 \%$ of our study group were free of genital symptoms suggests this possibility. Furthermore, the usefulness of the careful, methodical examination is highlighted by the fact that 11 of the 12 patients free of symptoms, but with clinical vulval lesions, had histological confirmation of VLP.

The diagnosis of LP requires clinical and pathological correlations that satisfy strict criteria. Previous studies showed the necessity of a histological analysis in each case with clinical history and features suspicious of LP. Furthermore, strong inter- and intra-observer variability in the clinical and histological assessment of OLP, based on the World Health Organization (WHO) definition, has been reported. ${ }^{11,12}$ The WHO criteria were revised by van der Meij and van der Waal in order to obtain a more reproducible diagnosis of OLP. We slightly modified their approach, ${ }^{13}$ extending the criteria also to cases of VLP.

DIV was considered to be 'LP in disguise'. ${ }^{14}$ Pelisse et al. proposed that all forms of DIV were LP presenting as part of the VVGS. ${ }^{15}$ To date, no agreement has been reached regarding histological patterns of this inflammatory vaginitis. ${ }^{16} \mathrm{We}$ found DIV to be an inflammatory condition strongly associated with genital LP even in the absence of vaginal LP lesions.

To the best of our knowledge, this is the first study addressing histologically confirmed VLP in a selected OLP cohort. During a brief study period we found a higher than expected prevalence of VLP $(57 \%)$, with a high rate $(92 \%)$ of VLP in symptomless patients. We conclude that this genital dermatosis has been underestimated or not recognized in the past. We believe that coordination among gynaecologists, oral medicine practitioners and pathologists, together with methodological accuracy, ${ }^{17,18}$ enabled us to achieve a wider awareness of the prevalence of LP.

\section{References}

1 Lewis FM. Vulval lichen planus. Br J Dermatol 1998; 138:569-75.

2 Lynch PJ, Edwards L. Genital Dermatology. New York: Churchill Livingstone, 1994; 95.

3 Boyd AS, Neldner K. Lichen planus. J Am Acad Dermatol 1991; 25:593-619.

4 Micheletti L, Preti M, Bogliatto F et al. Vulval lichen planus in the practice of a vulval clinic. Br J Dermatol 2000; 143:1349-50.

5 Pelisse M, Leibowitch M, Sedel D, Hewitt J. Un nouveau syndrome vulvo-vaginal gingival: lichen planus érosif plurimuqueux. Ann Dermatol Venereol 1982; 109:797-8.

6 Rogers RS, Eisen D. Erosive oral lichen planus with genital lesions. The vulvo vaginal-gingival syndrome and the peno-gingival syndrome. Dermatol Clin 2003; 21:91-8.

7 Eisenberg E. Oral lichen planus: a benign lesion. Oral Maxillofac Surg 2000; 58:1278-85.

8 Eisen D. The clinical manifestations and treatment of oral lichen planus. Dermatol Clin 2003; 21:79-89.

9 Eisen D. The evaluation of cutaneous, genital, scalp, nail, esophageal and ocular involvement in patients with oral lichen planus. Oral Surg Oral Med Oral Pathol Oral Radiol Endod 1999; 88:431-6.

10 Eisen D. The clinical features, malignant potential and systemic associations of oral lichen planus: a study of 723 patients. J Am Acad Dermatol 2002; 46:207-14.

11 van der Meij EH, Reibel J, Slootweg PJ. Interobserver and intraobserver variability in the histologic assessment of oral lichen planus. J Oral Pathol Med 1999; 28:274-7.

12 van der Meij EH, Schepman PK, Plonait DR et al. Interobserver and intraobserver variability in the clinical assessment of oral lichen planus. J Oral Pathol Med 2002; 31:95-8.

13 van der Meij EH, van der Waal I. Lack of clinicopathologic correlation in the diagnosis of oral lichen planus based on the presently available diagnostic criteria and suggestion for modifications. J Oral Pathol Med 2003; 32:507-12.

14 Edwards L, Friedrich EG. Desquamative vaginitis: lichen planus in disguise. Obstet Gynecol 1988; 71:832-6.

15 Pelisse M. Erosive vulvar lichen planus and desquamative vaginitis. Semin Dermatol 1996; 15:47-50.

16 Murphy R. Desquamative inflammatory vaginitis. Dermatol Ther 2004; 17:47-9.

17 Micheletti L, Preti M, Bogliatto F et al. Vulvology. A proposal for a multidisciplinary subspecialty. J Reprod Med 2002; 47:715-17.

18 Moyal-Barracco M, Edwards L. Diagnosis and therapy of anogenital lichen planus. Dermatol Ther 2004, 17:38-46. 